\title{
Analysis by finite element method of dynamic characteristics of the external ear canal in neonates
}

\author{
Michio MURAKOSHI*, Shohei TAKEDA** and Hiroshi WADA*** \\ * Department of Mechanical Engineering, Kagoshima University \\ 1-21-40 Korimoto, Kagoshima 890-0065, Japan \\ ** Department of Bioengineering and Robotics, Tohoku University \\ 6-6-01 Aoba-yama, Sendai 980-8579, Japan \\ *** Department of Intelligent Information Systems, Faculty of Science and Technology, Tohoku Bunka Gakuen University \\ 6-45-1 Kunimi, Sendai 981-8551, Japan \\ E-mail: wada@ait.tbgu.ac.jp
}

Received: 31 October 2016; Revised: 19 February 2017; Accepted: 2 March 2017

\begin{abstract}
Although early diagnosis and treatment of hearing disorders in neonates is highly effective for realization of linguistic competence and intellectual development, the diagnostic accuracy in the initial period of life is low. To overcome this problem, extensive research studies to diagnose the neonatal hearing with tympanometry, multifrequency tympanometry, the wideband acoustic transfer function technique, the sweep frequency impedance (SFI) technique and others have been conducted. However, since the results obtained are not consistent with those obtained in adults/children, diagnosis of hearing in neonates has been problematic. The difference in the dynamic characteristics of the hearing apparatus between adults/children and neonates, especially due to the morphological difference in the external ear canal, has been suggested as a possible reason for such problem. However, the dynamics of the neonatal hearing system has not yet been clarified. In the present study, therefore, three types of finite-element models of the neonatal external ear were developed and their dynamic characteristics (i.e., natural frequencies and mode shapes) were investigated. Frequency analyses of the constructed models revealed that the neonatal external ear canal wall has a resonance of around $0.3 \mathrm{kHz}$ and that this resonance leads to the volume change of such canal. This result suggests that the neonatal external ear canal wall may exhibit intrinsic oscillatory behavior if conventional tympanometry with a probe tone of $226 \mathrm{~Hz}$ is performed, affecting the diagnostic data in neonates.
\end{abstract}

Key words : Dynamic characteristics, External ear, Middle ear, Neonates, Finite element model

\section{Introduction}

Hearing impairment is one of the most common disorders at birth, i.e., about $0.1 \%$ newborns suffer from such disorders (Kennedy and McCann, 2004; Mehl and Thomson, 2002; Morton and Nance, 2006; Norton et al., 2000; Watkin and Baldwin, 1999; Wessex Universal Neonatal Hearing Screening Trial Group, 1998). Although early diagnosis and treatment of such disorders in neonates is highly effective for realization of linguistic competence and intellectual development (Yoshinaga-Itano et al., 1998), diagnostic accuracy during the initial neonatal period of the birth is low.

Tympanometry has generally been used for the diagnosis of middle ear dysfunction. The $226-\mathrm{Hz}$ probe tone at $85 \mathrm{~dB}$ SPL is presented to the ear canal by an earphone and the acoustic admittance is measured by a microphone while the static pressure of the ear canal is changed from $200 \mathrm{daPa}$ to $-400 \mathrm{daPa}$. A graph obtained by this method is called tympanogram. The horizontal and vertical axes of the tympanogram indicate the static pressure and acoustic admittance, respectively. The classification system of tympanograms was developed by Liden (1969) and Jerger (1970). From the normal middle ear, a pointed tympanogram with a sharp peak at around $0 \mathrm{daPa}$, referred as Type $\mathrm{A}$, is obtained. In contrast, a flat 

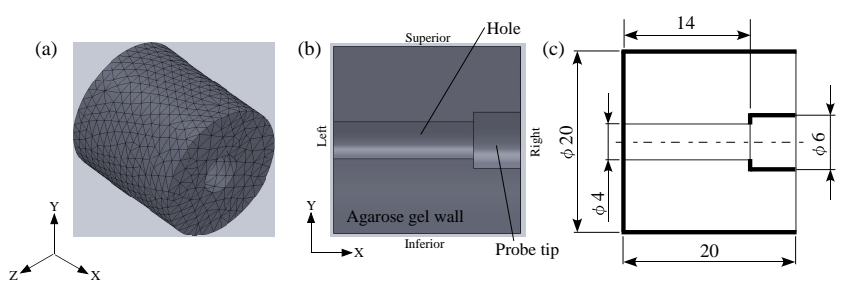

Fig. 1 Model 1 representing the gel model. (a) Three-dimensional view. (b) Cross-sectional view of X-Y plane. (c) Boundary conditions. The thick and thin lines show the fixed and free planes, respectively.
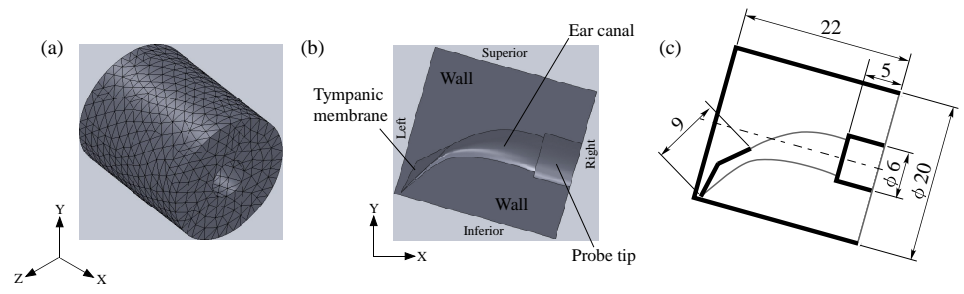

Fig. 2 Model 2 representing the neonatal external ear canal with a uniform Young's modulus. (a) Threedimensional view. (b) Cross-sectional view of X-Y plane. (c) Boundary conditions. The thick and thin lines show the fixed and free planes, respectively.

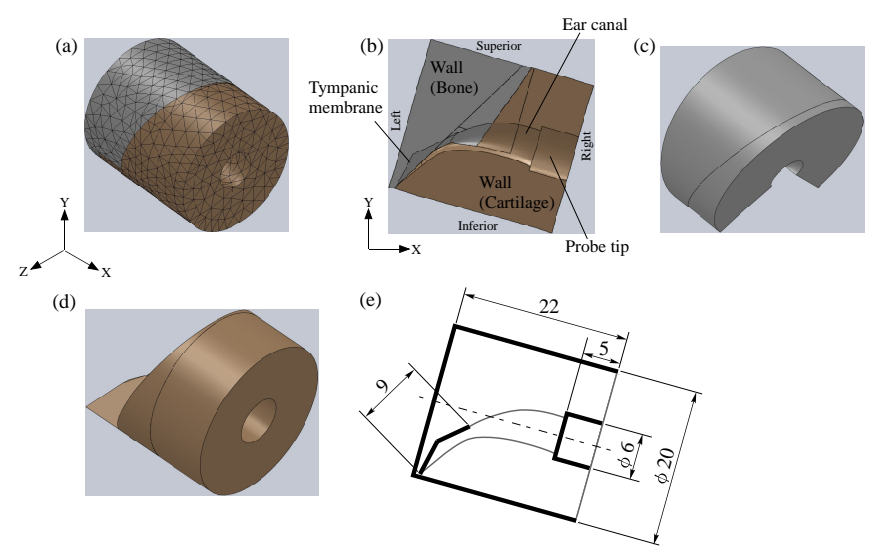

Fig. 3 Model 3 representing the neonatal external ear canal with two different Young's moduli of the bony cartilaginous structures. (a) Three-dimensional view. (b) Cross-sectional view of X-Y plane. (c) The bony structure (gray). (d) The cartilage (brown). (e) Boundary conditions. The thick and thin lines show the fixed and free planes, respectively.

tympanogram, Type B, is measured from the ear with fluid or infection behind the eardrum such as secretory otitis media. Conventional 226-Hz tympanometry has been acknowledged as a reliable method for detecting middle ear dysfunction in adults and children.

Results of conventional tympanometry with a probe tone of $226 \mathrm{~Hz}$ revealed that type-A tympanograms were obtained from neonates with middle ear effusion (Meyer et al., 1997; Paradise et al., 1976; Shurin et al., 1976). In other cases, neonates with a normal otoscopic finding have been reported to show type-B tympanograms (Keefe and Levi, 1996; Wright et al., 1985) or multi-peaked tympanograms (Keith, 1973; Himelfarb et al., 1979). Multi-frequency tympanometry, developed in the 1970s by Colletti $(1975,1976,1977)$, has also been applied to neonates (Holte et al., 1991; Keefe et al., 1993; Meyer et al., 1997), and two possible resonances at higher (about $1 \mathrm{kHz}$ ) and lower $(300 \mathrm{~Hz} / 450 \mathrm{~Hz})$ frequencies were suggested although such is not in adults and children. In a study using the wideband acoustic transfer function technique, which includes energy reflectance and acoustic admittance, significant differences in absorbance were identified between infants and adults across a wide frequency range $(0.125-10.0 \mathrm{kHz})$, the greatest difference occurring below $1 \mathrm{kHz}$ (Keefe et al., 1993). In our previous studies, the sweep frequency impedance (SFI) meter was developed to measure the dynamic characteristics of the middle ear (Wada et al., 1989, Wada et al., 1990). It measures sound pressure level (SPL) in the external ear canal when a sound of the sweeping sinusoidal frequency between $0.1 \mathrm{kHz}$ and $2.0 \mathrm{kHz}$ is delivered into this canal. It has been successfully used to detect middle ear problems in adults/children (Wada et al., 
1998). To extend its versatility, the SFI meter has been redesigned, and SFI tests were performed in neonates. As a result, the SPL was found to show two variations, one at around $0.3 \mathrm{kHz}$ and the other at around $1.2 \mathrm{kHz}$ (Aithal et al., 2016a, 2016b, 2015, 2014; Murakoshi et al., 2013). Since the SPL shows a variation at $1.3 \pm 0.3 \mathrm{kHz}$ due to the resonance of the middle ear in normal-hearing adults and children (Zhao et al., 2002), the variation found at around $1.3 \mathrm{kHz}$ is probably related to such resonance in neonates. However, the source of the variation at around $0.3 \mathrm{kHz}$ is still unclear.

The dynamic behavior of the hearing apparatus in neonates has been suggested to differ from that in adults and children (Keefe and Levi, 1996; Meyer et al., 1997; Paradise et al., 1976; Williams et al., 1995). Such difference has been speculated to be due to anatomical differences of the hearing system caused by postnatal development (Aithal et al., 2016, 2015, 2014; Himelfarb et al., 1979; Holte et al., 1991; Keefe, et al., 1993; Keith, 1975; Meyer et al., 1997; Murakoshi et al., 2013; Paradise et al., 1976). Possible components contributing to the dynamic behavior of the neonatal hearing apparatus include the external and middle ears. As for the middle ear, Kirikae (1960) performed a careful morphological investigation of the ossicles of different ages from birth to 81 years of age and found the following characteristics: the dimension of the ossicle does not show significant differences by age and the mass of the ossicle in neonates is 0.8 times smaller than that in adults. This implies that the angular stiffness of the neonatal ossicle may possibly be smaller than that of adults to the same extent, and that the difference in the resonance frequency of the middle ear in adults/children and neonates is limited to a few hundred-Hertz range at around $1 \mathrm{kHz}$. In contrast, the external ear canal is not completely formed until about 1 year of age (Anson et al., 1955; Anson and Donaldson, 1981). The neonatal external ear canal is about 50\% shorter in length and diameter than that of adults, which means that the ear canal volume in neonates is about one-tenth of that of adults (Keefe and Levi, 1996; McLellan and Webb, 1957; Noh and Lee, 2012; Saunders et al., 1983; Qi et al., 2006). In addition to the differences in dimensions, the mechanical properties of the cartilage surrounding the external ear canal changes in an age-dependent manner although details of such changes have not yet been reported.

In the present study, therefore, to explore the possibility that the neonatal external ear canal oscillates at around 0.3 $\mathrm{kHz}$, the dynamic characteristics (i.e., natural frequencies and mode shapes) of this canal were investigated.

\section{Materials and methods}

\subsection{Analytical models}

To elucidate the dynamic characteristics of the neonatal external ear canal, three finite-element models with tetrahedral solid elements were developed and frequency analyses were performed using a commercial finite-element method (FEM) program, SolidWorks 2010 (Dassault Systems SolidWorks Corp., MA). One was a finite-element model representing the gel model of a simplified neonatal external ear canal (Model 1), which was developed in our previous study (Murakoshi et al., 2013), and the others were finite-element models representing that canal (Model 2 and Model 3). The external ear canal wall of Model 2 was equipped with a uniform Young's modulus, whereas that of Model 3 was more precisely modeled, i.e., the wall was divided into a soft cartilaginous part and a hard bony part.

\subsection{Geometry and boundary conditions}

The geometry and boundary conditions of Model 1 are shown in Fig. 1. The geometry of Model 1 is based on that of the gel model developed in our previous study (Murakoshi et al., 2013). The boundary conditions of Model 1 are the same as those of SFI tests in the gel models, i.e., the inner wall and lateral side of Model 1 are free and the others are fixed.

The geometry and boundary conditions of Model 2 and Model 3 are shown in Figs. 2 and 3, respectively. The geometry of Model 2 is based on the anatomy of the neonatal external ear canal and tympanic membrane (Table 1). In this study, the inner diameter, outer diameter, roof length and floor length of the models are $1.6 \mathrm{~mm}$ to $4.8 \mathrm{~mm}, 20 \mathrm{~mm}, 16 \mathrm{~mm}$

Table 1 Dimensions of the external ear canal and tympanic membrane.

\begin{tabular}{lrr}
\hline & Adults & Neonates \\
\hline External ear canal & $9.0^{a}$ & $1.6-4.8^{c}$ \\
Inner diameter (mm) & $20^{c}$ & $1.6-4.8^{c}$ \\
Outer diameter (mm) & $20^{c}$ & $13-22.5^{c}$ \\
Roof length (mm) & $25-30^{b}$ & $17-22.5^{c}$ \\
Floor length (mm) & $25-30^{b}$ & \\
Tympanic membrane & $4.38^{d}$ & $4.38^{d}$ \\
Radius along the manubrium (mm) & $4.47^{d}$ & $4.47^{d}$ \\
Radius perpendicular to the manubrium (mm) & $1.46^{d}$ & 4.5 \\
Height of the cone (mm) & $4.46^{d}$ \\
\hline
\end{tabular}

${ }^{a}$ Wada et al., 1990, ${ }^{b}$ Saunders et al., 1983, ${ }^{c}$ Qi et al., 2006, ${ }^{d}$ Sum et al., 2002. 
Table 2 Mechanical properties of each model.

\begin{tabular}{lcccc}
\hline & Model 1 & Model 2 & \multicolumn{2}{c}{ Model 3 } \\
\cline { 3 - 5 } & & & Temporal bone & Cartilage \\
\hline Young's modulus $(\mathrm{kPa})$ & 95.7 & 45 & $3.8 \times 10^{5} a$ & 45 \\
Density $\left(\mathrm{kg} / \mathrm{m}^{3}\right)$ & 1,080 & 1,080 & 1,080 & 1,080 \\
Poisson's ratio & 0.49 & 0.49 & 0.3 & 0.49 \\
\hline
\end{tabular}

${ }^{a}$ Gzik et al., 2009.

and $22.5 \mathrm{~mm}$, respectively. In addition, for the sake of simplicity, the tympanic membrane was assumed to be conical in shape. The radius and height of the cone of the tympanic membrane are $4.5 \mathrm{~mm}$ and $1.5 \mathrm{~mm}$, respectively. In this model, the tympanic membrane, the entrance of the ear canal contacted with the measurement probe and the outermost surface of the model are assumed to be fixed and the others are free.

Model 3 has the same geometry as Model 2. Since it has been reported that the temporal bone encloses the neonatal external ear canal except for the underside of tympanic membrane (Qi et al., 2006), Model 3 was developed by dividing Model 2 into two parts, i.e., the gray part representing the temporal bone and the brown part representing the cartilage. The boundary conditions of Model 3 are the same as those of Model 2.

\subsection{Mechanical properties of the models}

Mechanical properties of each model are summarized in Table 2. Young's modulus and the density of Model 1 were set to be the same as those of the gel model with a concentration of $1.0 \mathrm{wt} \%$, i.e., $95.7 \mathrm{kPa}$ and $1,080 \mathrm{~kg} / \mathrm{m}^{3}$, respectively. Poisson's ratio of Model 1 was 0.49 because the gel is incompressible. The number of nodes and elements are 17,120 and 11,442 , respectively.

Since Young's modulus of the elastic cartilage in adults has been reported to be between $100 \mathrm{kPa}$ and $1 \mathrm{MPa}$ and since this modulus has been reported to increase by about $275 \%$ from birth to adulthood, Young's modulus of the neonatal external ear canal wall could be assumed to be between 36 and 364 kPa (Saunders et al., 1983; Qi et al., 2006). Young's modulus of Model 2 was assumed to be $45 \mathrm{kPa}$ in this study. The density and Poisson's ratio are the same as those of Model 1, i.e., $1,080 \mathrm{~kg} / \mathrm{m}^{3}$ and 0.49 , respectively. The number of nodes and elements are 25,777 and 17,261, respectively.

Young's modulus of the skull bone of 3-month old children has been reported to be about $380 \mathrm{MPa}$ (Gzik et al., 2009). In Model 3, therefore, this Young's modulus was applied to the temporal bone (gray part; Fig.3). Poisson's ratio of the gray part is assumed to be 0.3 because the bone is compressible. On the other hand, Young's modulus and Poisson's ratio of the cartilage (brown part; Fig. 3) were set to be the same as those of Model 2, i.e., $45 \mathrm{kPa}$ and 0.49 , respectively. Densities of both parts were assumed to be the same as those of Model 1 and Model 2, i.e., $1,080 \mathrm{~kg} / \mathrm{m}^{3}$. The number of nodes and elements are 22,002 and 14,534, respectively.

\subsection{Frequency analysis}

Frequency analysis of the developed models was performed in the commercial FEM environment SolidWorks Sim-

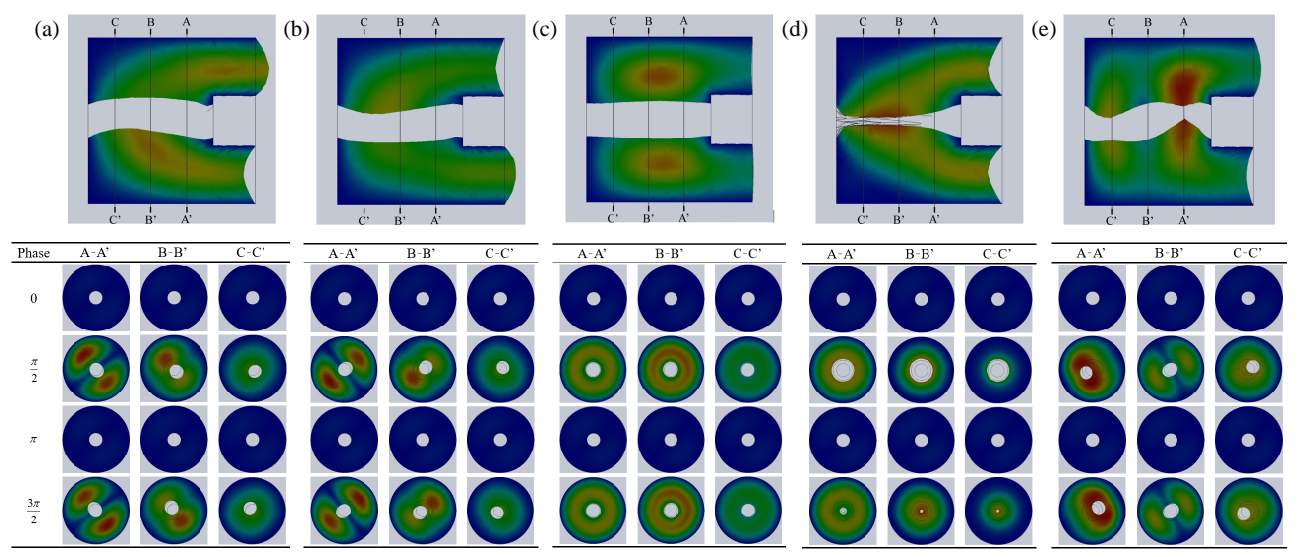

Fig. 4 Displacement distribution of Model 1. (a) First mode. (b) Second mode. (c) Third mode. (d) Fourth mode. (e) Fifth mode. Upper panels show a cross-sectional view of the X-Y plane at the phase of $\pi / 2$ and lower panels show cross-sectional view of the $\mathrm{Y}-\mathrm{Z}$ plane in different phases. Cross sections were obtained in three planes (A-A', B-B' and C-C'). 


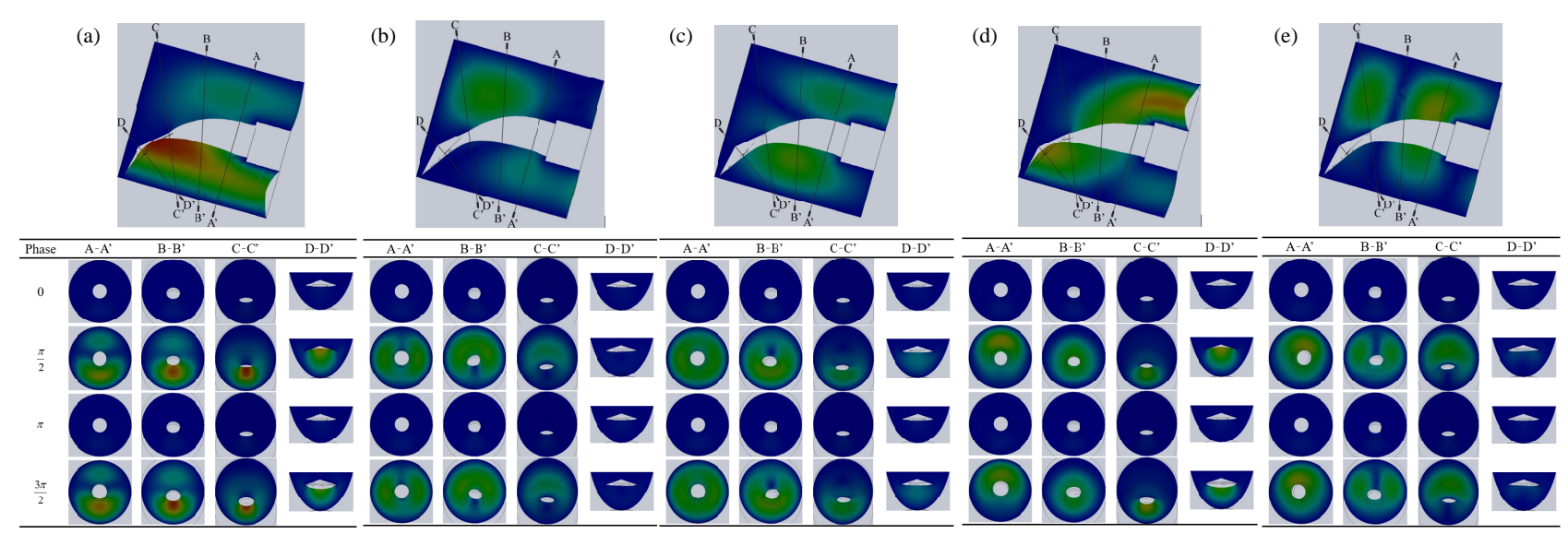

Fig. 5 Displacement distribution of Model 2. (a) First mode. (b) Second mode. (c) Third mode. (d) Fourth mode. (e) Fifth mode. Upper panels show cross-sectional view of the X-Y plane at the phase of $\pi / 2$ and lower panels show cross-sectional view of the $\mathrm{Y}-\mathrm{Z}$ plane in different phases. Cross sections were obtained in four planes (A-A', B-B', C-C' and D-D').

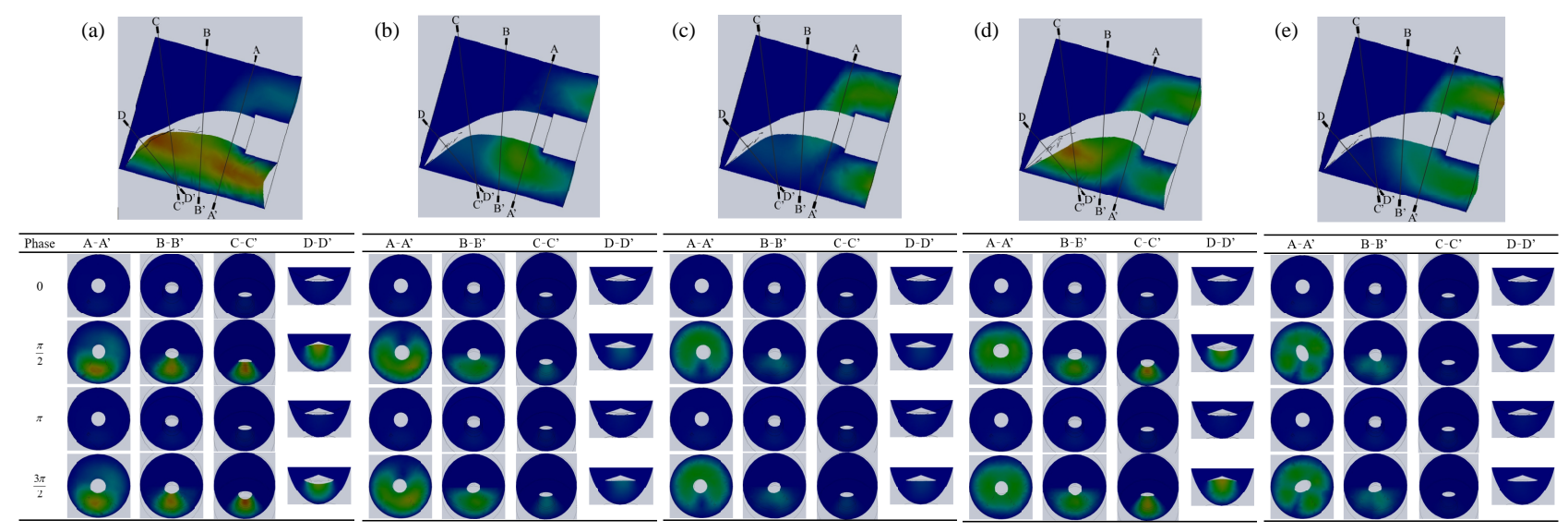

Fig. 6 Displacement distribution of Model 3. (a) First mode. (b) Second mode. (c) Third mode. (d) Fourth mode. (e) Fifth mode. Upper panels show cross-sectional view of the X-Y plane at the phase of $\pi / 2$ and lower panels show cross-sectional view of the $\mathrm{Y}-\mathrm{Z}$ plane in different phases. Cross sections were obtained in four planes (A-A', B-B', C-C' and D-D').

ulation 2010 (Dassault Systems SolidWorks Corp.). The first five undamped natural frequencies were analyzed without any load applied and compared for each model.

\section{Results}

\subsection{Frequency analysis of a finite-element model representing the gel model (Model 1)}

Figure 4 shows the calculated oscillation modes (from first to fifth) of Model 1. In Figs. 4(a) and 4(b), the first and second modes showed that the roof and floor walls moved like the first mode of a beam. They also moved in the same phase. Thus, the volume change of the ear canal was small. In Fig. 4(c), the third mode showed a twisting motion of the inner wall around the central axis of the ear canal. In Fig. 4(d), the fourth mode showed the bending motion of the inner wall similar to those of the first and second modes. However, the roof and floor walls moved in the opposite phase, resulting in a large volume change in the canal. In Fig. 4(e), the fifth mode showed that the inner wall moved like the second mode of a beam. The resonance frequencies of these modes are summarized in Table 3 (second column from the left).

\subsection{Frequency analyses of finite-element models representing the neonatal external ear canal (Models 2 and 3)}

First, second, third, fourth and fifth modes of Model 2 were obtained as shown in Fig. 5. In the first mode (Fig. 5(a)), the movement of the roof wall was small, whereas the floor wall largely moved toward the tympanic membrane. Similar tendencies were found in the fourth mode (Fig. 5(d)). The other modes showed twisting motion, leading to a small change in the ear canal volume. The resonance frequencies of these modes were about $250 \mathrm{~Hz}$ (Table 3; third column from the left). 
Table 3 Resonance frequencies of the models in each mode.

\begin{tabular}{lccr}
\hline \multirow{2}{*}{ Mode } & & Resonance frequencies (Hz) & Model 3 \\
\cline { 2 - 4 } & Model 1 & Model 2 & 274.21 \\
1st & 365.48 & 246.04 & 275.71 \\
2nd & 365.76 & 247.76 & 290.56 \\
3rd & 366.92 & 253.70 & 292.10 \\
4th & 409.11 & 260.55 & 319.22 \\
5th & 415.37 & 276.41 & 2 \\
\hline
\end{tabular}

Table 4 Volume change in the ear canal in each mode. The increase or decrease column represents the ratio of the maximum or minimum volume of the ear canal to that in the initial phase (i.e., phase 0 ), respectively.

\begin{tabular}{|c|c|c|c|c|c|c|}
\hline \multirow{2}{*}{ Mode } & \multicolumn{2}{|c|}{ Model 1} & \multicolumn{2}{|c|}{ Model 2} & \multicolumn{2}{|c|}{ Model 3} \\
\hline & increase $(\%)$ & decrease $(\%)$ & increase $(\%)$ & decrease $(\%)$ & increase $(\%)$ & decrease $(\%)$ \\
\hline $1 \mathrm{st}$ & 3 & 0 & 58 & 39 & 32 & 34 \\
\hline $2 \mathrm{nd}$ & 1 & 0 & 12 & 0 & 8 & 10 \\
\hline 3rd & 16 & 0 & 13 & 0 & 3 & 6 \\
\hline 4th & 183 & 87 & 58 & 40 & 25 & 28 \\
\hline 5 th & 7 & 3 & 11 & 0 & 0 & 4 \\
\hline
\end{tabular}

Figure 6 shows the first to fifth modes of Model 3. The tendencies of the movement of the ear canal wall were similar to those found in Model 2, that is, although the volume of the ear canal largely changed in the first and fourth modes, such changes were small in the other modes. The resonance frequencies of these modes are summarized in Table 3 (fourth column from the left).

\section{Discussion}

\subsection{Dynamic characteristics of the gel model (Model 1)}

In the present study, a finite-element model representing the $1.0 \mathrm{w} \%$ gel model (Murakoshi et al., 2013) was developed. The calculated ratios of increase and decrease in the volume of the external ear canal $S_{C}$ in each mode are summarized in Table 4. In Model 1, only the fourth mode showed a much larger volume change in the external ear canal than those of the other modes, suggesting that this mode is possibly related to the resonance found in the gel model.

The calculated resonance frequencies in each mode ranged from $365.48 \mathrm{~Hz}$ to $415.37 \mathrm{~Hz}$ with an average frequency of about $385 \mathrm{~Hz}$ (Table 3), which is about 30\% smaller than that measured in the gel model in our previous study (Murakoshi et al., 2013), i.e., about $550 \mathrm{~Hz}$. Although the exact reasons for this discrepancy are unclear, dehydration of the gel during the measurement process is one possibility, leading to a larger Young's modulus of the gel and increased resonance frequency.

\subsection{Dynamic characteristics of the neonatal external ear canal (Models 2 and 3)}

The resonance frequencies calculated from Model 2 in each oscillating mode ranged from $246.04 \mathrm{~Hz}$ to 276.41 $\mathrm{Hz}$ with an average frequency of about $257 \mathrm{~Hz}$ (Table 3). The resonance frequencies calculated from Model 3 in each oscillating mode ranged from $274.21 \mathrm{~Hz}$ to $319.22 \mathrm{~Hz}$ with an average frequency of about $290 \mathrm{~Hz}$ (Table 3). These frequencies fell within the range of the low-frequency resonance found in neonates, i.e., about 200-450 Hz (Aithal et al., 2016a, 2016b, 2015, 2014; Holte et al., 1991; Keefe et al., 1993; Meyer et al., 1997; Murakoshi et al. 2013).

As is the case of Model 1, to identify a possible oscillating mode of the external ear canal in neonates, oscillating modes of Model 2 and Model 3 were evaluated in terms of the variation of the ear canal volume. The calculated ratios of the increase and decrease in each mode are summarized in Table 4.

As summarized in this table, the ratios of the ear canal volume in the first and fourth modes were much larger than those of the other modes in both models, suggesting that these modes are possibly predominant in the neonatal hearing apparatus. As shown in Fig. 6, a large movement of the inner wall underneath the tympanic membrane was found in these modes. In a previous study, a newborn ear-canal volume changes under a high static pressure was investigated using its finite-element model and the maximum displacement of the entire model was found to occur on the inferior surface of the external ear canal (Qi et al., 2006). Furthermore, the neonatal external ear canal could collapse under large negative pressure (Holte et al., 1990). Based on these results, it can be reasonably explained that the neonatal external ear canal shows intrinsic oscillatory behavior in the combination of the first and fourth modes represented by Model 2 and Model 3 , possibly being related to the low-frequency resonance reported in previous studies with various diagnostic techniques (Aithal et al., 2016, 2015, 2014; Holte et al., 1991; Keefe et al., 1993; Meyer et al., 1997; Murakoshi et al., 2013). 


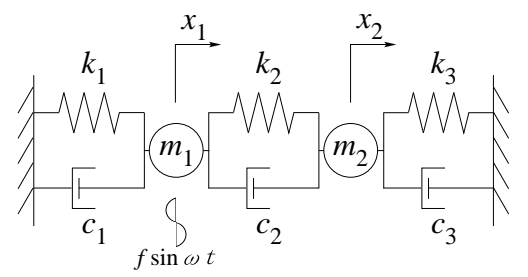

Fig. 7 Forced vibrations of a system with two degrees of freedom representing the neonatal external ear canal.

Regarding these resonance frequencies, those of Model 3 were larger than those of Model 2 due to the stiffer structure of the bony temporal bones.

\subsection{Analytical model of the external ear canal in neonates}

4.3.1. Forced vibrations of a system with two degrees of freedom In this study, the dumping coefficient was not considered when the finite-element models (Models 1-3) were analyzed. To analyze the first and fourth modes in Model 3 theoretically, therefore, a system with two degrees of freedom representing the neonatal external ear canal wall was constructed, as shown in Fig. 7. Since the external ear canal wall is surrounded by a bony structure and connected to it, both sides of such model were assumed to be fixed. Equations of motion of the system are expressed by

$$
\begin{aligned}
& m_{1} \frac{d^{2} x_{1}(t)}{d t^{2}}+\left(c_{1}+c_{2}\right) \frac{d x_{1}(t)}{d t}+\left(k_{1}+k_{2}\right) x_{1}-c_{2} \frac{d x_{2}(t)}{d t}-k_{2} x_{2}=f \sin \omega t \\
& m_{2} \frac{d^{2} x_{2}(t)}{d t^{2}}+\left(c_{2}+c_{3}\right) \frac{d x_{2}(t)}{d t}+\left(k_{2}+k_{3}\right) x_{2}-c_{2} \frac{d x_{1}(t)}{d t}-k_{2} x_{1}=0
\end{aligned}
$$

where $m_{1}$ and $m_{2}$ are mass, $k_{1}, k_{2}$ and $k_{3}$ are spring constants and $c_{1}, c_{2}$ and $c_{3}$ are damping coefficients of the system. $x_{1}(t)$ and $x_{2}(t)$ are the displacements of the mass $m_{1}$ and $m_{2}$ from their static equilibrium position at time $t$, respectively. A force $F(t)=f \sin \omega t$ is assumed to act on the mass $m_{1}$. $\omega$ is the angular frequency of the stimulus.

4.3.2. Determination of masses and spring constants When $c_{1}, c_{2}$ and $c_{3}$ were assumed to be zero, $m_{1}, m_{2}, k_{1}, k_{2}$ and $k_{3}$ were determined so that the two resonance frequencies of the system were close to those of first and fourth modes in Model 3, i.e., around $274 \mathrm{~Hz}$ and $292 \mathrm{~Hz}$. According to Eq. (1), equations of motion in this condition are expressed by

$$
\begin{aligned}
& m_{1} \frac{d^{2} x_{1}(t)}{d t^{2}}+\left(k_{1}+k_{2}\right) x_{1}-k_{2} x_{2}=f \sin \omega t \\
& m_{2} \frac{d^{2} x_{2}(t)}{d t^{2}}+\left(k_{2}+k_{3}\right) x_{2}-k_{2} x_{1}=0
\end{aligned}
$$

Therefore, the resonance frequencies of the system $f_{1}$ and $f_{2}\left(f_{1}<f_{2}\right)$ are expressed by

$$
\begin{aligned}
& f_{1}=\frac{1}{2 \pi} \sqrt{\frac{m_{1}\left(k_{2}+k_{3}\right)+m_{2}\left(k_{1}+k_{2}\right)-\sqrt{\left\{m_{1}\left(k_{2}+k_{3}\right)+m_{2}\left(k_{1}+k_{2}\right)\right\}^{2}-4 m_{1} m_{2}\left(k_{1} k_{2}+k_{2} k_{3}+k_{3} k_{1}\right)}}{2 m_{1} m_{2}}} \\
& f_{2}=\frac{1}{2 \pi} \sqrt{\frac{m_{1}\left(k_{2}+k_{3}\right)+m_{2}\left(k_{1}+k_{2}\right)+\sqrt{\left\{m_{1}\left(k_{2}+k_{3}\right)+m_{2}\left(k_{1}+k_{2}\right)\right\}^{2}-4 m_{1} m_{2}\left(k_{1} k_{2}+k_{2} k_{3}+k_{3} k_{1}\right)}}{2 m_{1} m_{2}}}
\end{aligned}
$$

Hereafter, $X_{1}$ and $X_{s t}$ express the amplitude and static displacement of $x_{1}$, respectively. According to Eq. (2), the relationship between the amplitude magnification $X_{1} / X_{s t}$ and frequencies $f$ is expressed by

$$
\frac{X_{1}}{X_{s t}}=\frac{k_{1}\left(k_{2}+k_{3}-m_{2} \omega\right)}{\left(k_{1}+k_{2}-m_{1} \omega\right)\left(k_{2}+k_{3}-m_{2} \omega\right)-k_{2}^{2}}
$$

The sum of $m_{1}$ and $m_{2}$ was determined to be $7.0 \times 10^{-3} \mathrm{~kg}$, which was obtained by rounding off the mass of Model $3\left(7.09 \times 10^{-3} \mathrm{~kg}\right)$ to the nearest whole number. Since the knowledge of the anatomical configuration of the external ear canal wall in neonates, including the morphology, the mechanical properties, the connection between different tissues and so on, is limited, the spring constants $k_{1}$ and $k_{3}$ are assumed to be similar to those of the cartilage (several tens of $\mathrm{kN} / \mathrm{m}$ ) 


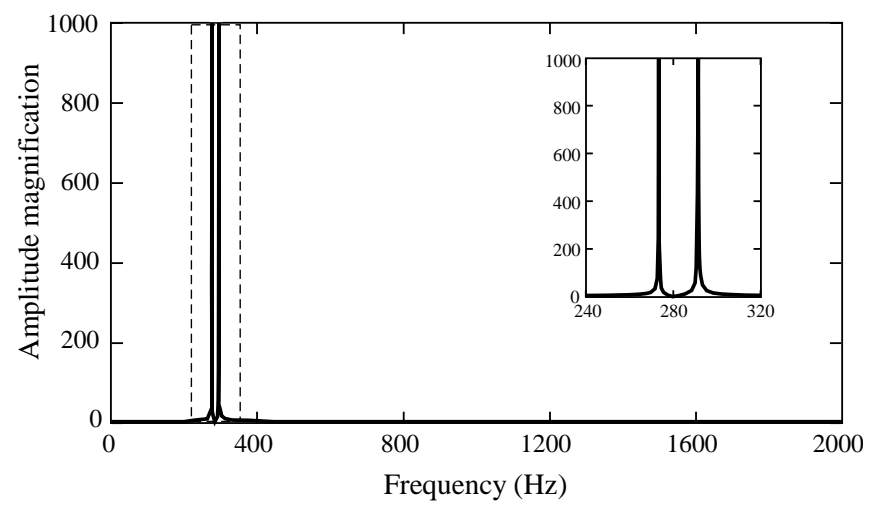

Fig. 8 Resonance curve of the mass $m_{1}$ when $c_{1}=c_{2}=c_{3}=0$. The inset indicates the magnification of the dotted box area.

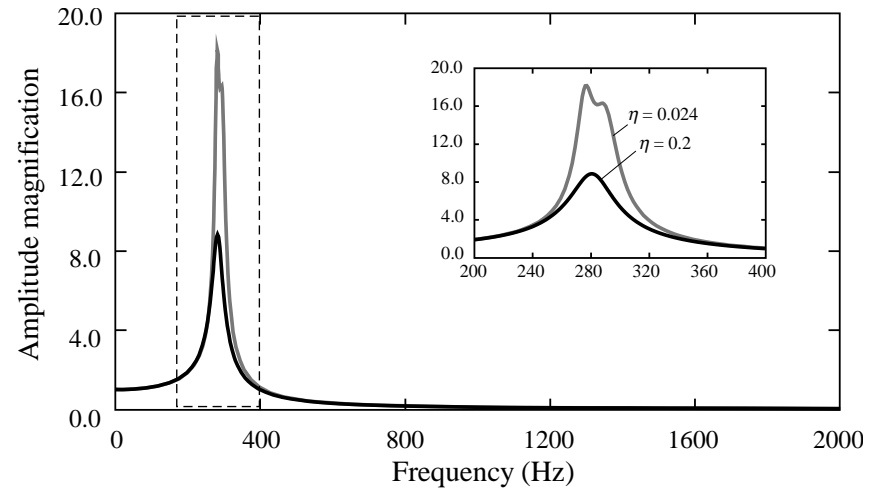

Fig. 9 Resonance curve of the mass $m_{1}$ with different values of $C$. The gray and black lines show such curves with $C=0.09(\eta=0.024)$ and $C=0.69(\eta=0.2)$, respectively. The inset indicates the magnification of the dotted box area.

and the tendon (several kN/m) (Ide and Amano, 1992; Kiriyama and Yamazaki, 2004), i.e., $18.3 \mathrm{kN} / \mathrm{m}$ and $3.12 \mathrm{kN} / \mathrm{m}$, respectively. The remaining spring constants $k_{2}$ and $m_{1}$ and $m_{2}$ were determined to be $0.51 \mathrm{kN} / \mathrm{m}, 5.83 \times 10^{-3} \mathrm{~kg}$ and $1.17 \times 10^{-3} \mathrm{~kg}$, respectively, so that the resonance frequencies of this model with two degrees of freedom corresponds to those obtained by the finite-element analysis, that is, $f_{1}$ becomes $274 \mathrm{~Hz}$ and $f_{2}$ becomes $292 \mathrm{~Hz}$. The response curve of mass $m_{1}$ is shown in Fig. 8. Two variations occurred at such resonance frequencies.

4.3.3. Determination of damping coefficients For the sake of simplicity, the damping coefficients $c_{1}, c_{2}$ and $c_{3}$ were assumed to be the same value, i.e., $c_{1}=c_{2}=c_{3}$. The damping coefficient was determined based on the damping ratio $\eta$. In the present study, $\eta$ was assumed to be expressed by the following equation:

$$
\eta=\frac{C}{2 \sqrt{M K}}
$$

where $C$ is the equivalent damping coefficient of the system, $M$ is the total mass of the system $\left(7.0 \times 10^{-3} \mathrm{~kg}\right)$ and $K$ is the equivalent spring constant of the system. The springs of the system are assumed to be connected in series. The equivalent spring constant of the system $K$ is therefore expressed by

$$
K=\frac{k_{1} k_{2} k_{3}}{k_{1} k_{2}+k_{2} k_{3}+k_{3} k_{1}} \cong 428
$$

In general, the damping ratio $\eta$ of biological materials ranges from 0.08 to 0.40 (Wada et al., 1992). In the present study, to indicate the effect of the damping ratio $\eta$ on the resonance curve, the response curves were calculated with two different equivalent damping coefficients $C$, i.e., one outside the range of the damping ratio of biological materials $(C=0.09 ; \eta=0.024)$ and the other within such range $(C=0.69 ; \eta=0.20)$.

The responses of the system are shown in Fig. 9. As shown in this figure, the two resonance peaks shown in Fig. 8 were found to be superimposed on each other. A superposition of two such peaks and a decrease in the amplitude were found when the damping coefficient increased. This is consistent with that obtained in the SFI measurement in neonates, that is, only one resonance was observed at about $0.3 \mathrm{kHz}$. These results indicate the possibility that the low-frequency 
resonance measured in neonates is a superposition of the first- and fourth-mode resonances of the external ear canal wall due to its damping effect.

\section{Conclusions}

In this study, to elucidate the dynamic characteristics (i.e., natural frequencies and mode shapes) of the neonatal external ear canal, analysis of the canal was performed using finite-element models. For this purpose, a finite-element model representing the gel model (Model 1) and finite-element models representing the neonatal external ear canal (Model 2 and Model 3) were developed and frequency analyses were performed. As a result, it was found that the external ear canal wall in neonates has a resonance frequency of around $0.3 \mathrm{kHz}$. This result suggests that the neonatal ear canal wall possibly shows intrinsic oscillatory behavior when conventional $226-\mathrm{Hz}$ tympanometry is performed, affecting the diagnostic data in neonates.

\section{Acknowledgements}

This work was supported by the Adaptable and Seamless Technology Transfer Program through Target-driven R\&D (A-STEP) 221Z010652400 and AS231Z02915F from the Japan Science and Technology Agency (JST) to H.W. and a grant from the Casio Science Promotion Foundation to M.M.

\section{References}

Aithal, V., Kei, J., Driscoll, C., Murakoshi, M. and Wada, H., Sweep frequency impedance measures in young infants: developmental characteristics from birth to 6 months, Int. J. Audiol., (2016a), in press.

Aithal, V., Kei, J., Driscoll, C., Murakoshi, M. and Wada, H., Effects of ear canal static pressure on the dynamic behaviour of outer and middle ear in newborns, Int. J. Pediatr. Otorhinolaryngol., Vol.82 (2016b), pp.64-72.

Aithal, V., Kei, J., Driscoll, C., Swanston, A., Murakoshi, M. and Wada, H., Sweep frequency impedance measures in Australian Aboriginal and Caucasian neonates, Int. J. Pediatr. Otorhinolaryngol., Vol.79 (2015), pp.1024-1029.

Aithal, V., Kei, J., Driscoll, C., Swanston, A., Roberts, K., Murakoshi, M. and Wada, H., Normative sweep frequency impedance measures in healthy neonates, J. Am. Acad. Audiol., Vol.25 (2014), pp.343-354.

Anson, B.J. and Donaldson, J.A., Surgical anatomy of the temporal bone and ear, Saunders, (1981).

Anson, B.J., Bast, T.H. and Richany, S.F., The fetal and early postnatal development of the tympanic ring and related structures in man, Ann. Otol. Rhinol. Laryngol., Vol.64 (1955), pp.802-823.

Colletti, V., Methodologic observations on tympanometry with regard to the probe tone frequency, Acta. Otolaryngol., Vol.80 (1975), pp.54-60.

Colletti, V., Multifrequency tympanometry, Audiology, Vol.16 (1977), pp.278-287.

Colletti, V., Tympanomety from 200 to $2000 \mathrm{~Hz}$ probe tone., Audiology, Vol.15 (1976), pp.106-119.

Gzik, M., Wolański, W., Tejszerska, D., Gzik-Zroska, B., Koźlak, M. and Larysz, D., Interdiciplinary researches supporting neurosurgical correction of children head deformation, Modelling and optimization of physical systems, Vol.8 (2009), pp.49-54.

Himelfarb, M.Z., Popelka, G.R. and Shanon, E., Tympanometry in normal neonates, J. Speech. Hear. Res., Vol.22 (1979), pp.179-191.

Holte, L., Cavanaugh, R.M. Jr and Margolis, R.H., Ear canal wall mobility and tympanometric shape in young infants, J. Pediatr., Vol.117 (1990), pp.77-80.

Holte, L., Margolis, R.H. and Cavanaugh, R.M. Jr, Developmental changes in multifrequency tympanograms, Audiology, Vol.30 (1991), pp.1-24.

Ide, T. and Amano, R., Stress analysis of the hip, Bulletin of Yamanashi Medical University, Vol.9 (1992), pp.69-75.

Jerger, J.F., Clinical experience with impedence audiometry, Arch. Otolaryngol., Vol.92 (1970), pp.311-324.

Keefe, D.H. and Levi, E., Maturation of the middle and external ears: acoustic power-based responses and reflectance tympanometry, Ear. Hear., Vol.17 (1996), pp.361-373.

Keefe, D.H., Bulen, J.C., Arehart, K.H. and Burns, E.M., Ear-canal impedance and reflection coefficient in human infants and adults, J. Acoust. Soc. Am., Vol.94 (1993), pp.2617-2638.

Keith, R., Middle ear function in neonates, Arch. Otolaryngol., Vol.101 (1975), pp.376-378.

Keith, R.W., Impedance audiometry with neonates, Arch. Otolaryngol., Vol.97 (1973), pp.465-467. 
Kennedy, C. and McCann, D., Universal neonatal hearing screening moving from evidence to practice, Arch. Dis. Child. Fetal. Neonatal. Ed., Vol.89 (2004), pp.F378-383.

Kirikae, I., The structure and function of the middle ear, The University of Tokyo Press, Tokyo, (1960).

Kiriyama, Y. and Yamazaki, N., Biomechanical analysis of spinal instruments applying rigid body and spring model, Biomechanisms, Vol.17 (2004), pp.185-194.

Liden, G., The scope and application of current audiometric tests, J. Laryngol. Otol. Vol.83 (1969), pp.507-520.

McLellan, M.S. and Webb, C.H., Ear studies in the newborn infant: Natural appearance and incidence of obscuring by vernix, cleansing of vernix, and description of drum and canal after cleansing, J. Pediatr., Vol.51 (1957), pp.672-677.

Mehl, A.L. and Thomson, V., The Colorado newborn hearing screening project, 1992-1999: on the threshold of effective population-based universal newborn hearing screening, Pediatrics, Vol.109 (2002), p.E7.

Meyer, S.E., Jardine, C.A. and Deverson, W., Developmental changes in tympanometry: a case study, Br. J. Audiol., Vol.31 (1997), pp.189-195.

Morton, C.C. and Nance, W.E., Newborn hearing screening-a silent revolution, N. Engl. J. Med., Vol.354 (2006), pp.21512164.

Murakoshi, M., Yoshida, N., Sugaya, M., Ogawa, Y., Hamanishi, S., Kiyokawa, H., Kakuta, R., Yamada, M., Takahashi, R., Tanigawara, S., Matsutani, S., Kobayashi, T. and Wada, H., Dynamic characteristics of the middle ear in neonates, Int. J. Pediatr. Otorhinolaryngol., Vol.77 (2013), pp.504-512.

Noh, H. and Lee, D.H., Direct measurement of ear canal volume in a pediatric population: Can we explain its individual variation in terms of age and body weight?, Int. J. Pediatr. Otorhinolaryngol., Vol.76 (2012), pp.658-662.

Norton, S.J., Gorga, M.P., Widen, J.E., Folsom, R.C., Sininger, Y., Cone-Wesson, B., Vohr, B.R., Mascher, K. and Fletcher, K., Identification of neonatal hearing impairment: evaluation of transient evoked otoacoustic emission, distortion product otoacoustic emission, and auditory brain stem response test performance, Ear. Hear., Vol.21 (2000), pp.508528.

Paradise, J.L., Smith, C.G. and Bluestone, C.D., Tympanometric detection of middle ear effusion in infants and young children, Pediatrics, Vol.58 (1976), pp.198-210.

Qi, L., Liu, H., Lutfy, J., Funnell, W.R. and Daniel, S.J., A nonlinear finite-element model of the newborn ear canal, J. Acoust. Soc. Am., Vol.120 (2006), pp.3789-3798.

Saunders, J.C., Kaltenbach, J.A. and Relkin, E.M., The structural and functional development of the outer and middle ear, Academic Press, New York, Vol. (1983), pp.3-25.

Shurin, P.A., Pelton, S.I. and Klein, J.O., Otitis media in the newborn infant, Ann. Otol. Rhinol. Laryngol., Vol.85 (1976), pp.216-222.

Sun, Q., Gan, R.Z., Chang, K.H. and Dormer, K.J., Computer-integrated finite element modeling of human middle ear, Biomech. Model. Mechanobiol., Vol.1 (2002), pp.109-122.

Wada, H. and Kobayashi, T., Dynamical behavior of middle ear: theoretical study corresponding to measurement results obtained by a newly developed measuring apparatus, J. Acoust. Soc. Am., Vol.87 (1990), pp.237-245.

Wada, H., Kobayashi, T., Suetake, M. and Tachizaki, H., Dynamic behavior of middle ear based on sweep frequency tympanometry, Audiology, Vol.28 (1989), pp.127-134.

Wada, H., Koike, T. and Kobayashi, T., Clinical applicability of the sweep frequency measuring apparatus for diagnosis of middle ear diseases, Ear. Hear., Vol.19 (1998), pp.240-249.

Wada, H., Metoki, T. and Kobayashi, T., Analysis of dynamic behavior of human middle ear using a finite-element method, J. Acoust. Soc. Am., Vol.92 (1992), pp.3157-3168.

Watkin, P.M. and Baldwin, M., Confirmation of deafness in infancy, Arch. Dis. Child., Vol.81 (1999), pp.380-389.

Wessex Universal Neonatal Hearing Screening Trial Group, Controlled trial of universal neonatal screening for early identification of permanent childhood hearing impairment, Lancet, Vol.352 (1998), pp.1957-1964.

Williams, M., Purdy, S. and Barber, C., High frequency probe tone tympanometry in infants with middle ear effusion, Aust. J. Otolaryng., Vol.2 (1995), pp.169-173.

Wright, P.F., McConnell, K.B., Thompson, J.M., Vaughn, W.K. and Sell, S.H., A longitudinal study of the detection of otitis media in the first two years of life, Int. J. Pediatr. Otorhinolaryngol., Vol.10 (1985), pp.245-252.

Yoshinaga-Itano, C., Sedey, A.L., Coulter, D.K. and Mehl, A.L., Language of early- and later-identified children with hearing loss, Pediatrics, Vol.102 (1998), pp.1161-1171.

Zhao, F., Wada, H., Koike, T., Ohyama, K., Kawase, T. and Stephens, D., Middle ear dynamic characteristics in patients with otosclerosis, Ear Hear. Vol.23 (2002), pp.150-158. 